\title{
Video moving target binary image processing method based on OTSU
}

\author{
Xiaowei Han ${ }^{1, \text { a }}$,Yuan $\mathrm{Gao}^{2, \mathrm{~b}}$,Yong $\mathrm{Cao}^{3, \mathrm{c}}$,Zheng $\mathrm{Lu}^{4, \mathrm{~d}}$,Dun Niu${ }^{5, \mathrm{e}}$ \\ ${ }^{1}$ College of Information Engineering Shenyang University \\ Liaoning Shenyang P.R. China \\ ${ }^{2}$ College of Information Engineering Shenyang University \\ Liaoning Shenyang P.R. China \\ ${ }^{3}$ College of Information Engineering Shenyang University \\ Liaoning Shenyang P.R. China \\ ${ }^{4}$ College of Information Engineering Shenyang University \\ Liaoning Shenyang P.R. China \\ ${ }^{5}$ North China Regional Air Traffic Management Bureau of CAAC, Hang An Road ,Beijing Capital
International Airport, Beijing, 100621, China \\ aHXW69@163.com, ${ }^{\mathrm{b}} 307400614 @ q q . c o m,{ }^{\mathrm{c}} 543530681 @ q q . c o m,{ }^{\mathrm{d}} 774324529 @ q q . c o m$, \\ eniudun1203@163.com
}

Keywords: Video image processing. Binary image. Gaussian Mixture Models(GMM). OTSU

\begin{abstract}
Because of some pixels of foreground image which is binarized by fixed threshold, the Gaussian mixture model can not get accurate result. So we introduce OTSU to look for the threshold which satisfy maximum-between-clusters-variance, and it will get best divisional effect.It achieve adaptive threshold image dynamic adjustment of image binarization. Then use video data to validate, the result shows that the algorithm have better result by comparing with the traditional binarization algorithm in the video image.
\end{abstract}

\section{Introduction}

There is a lot of binarization method of image, all kinds of method have merits and faults for themselves, the different methods will get the different results for the same image. Kittler ${ }^{[1]}$ and others put forward threshold method for selecting which combine gradient value of gray with gray statistics in image.The principle of Bernsen algorithm ${ }^{[2]}$ is to get the threshold of pixel with maximum-minimum pixels in the local window. Niblack ${ }^{[3]}$ algorithm based on the mean of neighborhood pixels of each pixel and variance of neighborhood pixels of each pixel to caculate the threshold of pixels. VFCM ${ }^{[4]}$ algorithm is binarization methods which combine with the method of global thresholding of maximal variance and the FCM method. NFCM algorithm ${ }^{[5]}$ is binarization methods which combine with the method of Niblack local threshold and the FCM method.

The gaussian mixture of pixel leve can effectively describe the multimodal background and can self-adapting change with dynamic background.It can achieve better result on motion target detection,so it has been widely paid much attention. Stauffe ${ }^{[6]}$ and Grimson use the adaptive gaussian mixture model to patch for every pixel with gaussian mixture distribution. And update the model by online estimation, it can reliably handle effect of illumination change and the interference of background chaos. Zhang and others ${ }^{[7]}$ judge pixels of foreground with SVM on GMM method, and it can reduce error rates of complicated environment detection. R. T. Colin and others ${ }^{[8-9]}$ establish a background model of single Gaussian. Shimada and others ${ }^{[10]}$ reduce amount of the original algorithm by removing Gaussian distribution of combined part.

Compared with other methods of threshold selection, we use OTSU method. Based on the definition of variance, it can be seen that the bigger variance between cluster constitute the bigger difference of foreground and background in image. If infra-class variance is small, it shows that the difference of infra-class part is small. As a result, the binary threshold which make variance between cluster biggest means get the error probability of pixel of background and foreground, so we will use 
adaptive threshold of OTSU to imptove the effect of binarization with the detection methods of background modeling which is Gaussian mixture model. The result shows that the algorithm have better result of the binarization that compare with the traditional binarization algorithm in the video image.

\section{Gaussian mixture model}

GMM(Gaussian mixture model) consider the color value of pixel in the background accord with Gaussian distribution, Gaussian distribution of multiple different weights simulate color changes of pixel.

The definition of model. The observations of pixel $(x, y)$ at time $t$ in video frame $I$ record $I_{t}$, a series of observation $\left\{I_{1}, I_{2}, \ldots, I_{t}\right\}$ at different times can be thought random process which independent of others, can use K Gaussian expression:

$$
M_{i, t}=M\left(\delta_{i, t}^{2}, \mu_{i, t}\right)
$$

Among them, $\mathrm{i}=1,2, \ldots, \mathrm{K}$. The value of $\mathrm{K}$ is determined by factors of computing performance, usually we will take 3 to 7 . The probability distribution of point $(\mathrm{x}, \mathrm{y})$ at time $\mathrm{t}$ is :

$$
P\left(I_{t}\right)=\sum_{i=1}^{K} w_{i, t} \eta\left(I_{t}, \Sigma_{i, t}, \mu_{i, t}\right)
$$

Among them,$w_{i, t}$ is weight of i-gaussian distribution at time t,it reflects proportion which gaussian distribution appears, and $\sum_{i=1}^{K} w_{i, t}=1 ; \eta\left(I_{t}, \Sigma_{i, t}, \mu_{i, t}\right)$ is probability density function whose first mean is $\mu_{i, t}$,covariance is $\Sigma_{i, t}$ at time t:

$$
\eta\left(I_{t}, \Sigma_{i, t}, \mu_{i, t}\right)=\frac{1}{(2 \pi)^{\frac{n}{2}}|\Sigma|^{\frac{1}{2}}} \exp \left(-\frac{1}{2}\left(I_{t}-\mu_{t}\right)^{T} \Sigma^{-1}\left(I_{t}-\mu_{t}\right)\right)
$$

Model update. If the current pixel values and mean of Gaussian distribution satisfy $\left|I_{t}-\mu_{i, t}\right| \leq D \delta_{i, t}^{2}$, we will consider the match is successful, and experience value of D is 2.5. If there are multiple matches, we will choose the best one. It will adjust weights of distribution when matching success:

$$
w_{i, t}=\alpha N_{i, t}+(1-\alpha) w_{i, t-1}
$$

Among them, $\alpha$ is learning rate, value is between 0 and 1 , the bigger $\alpha$ is ,the fast weight update,

and vice versa; $N_{i, t}$ is 1 for the distribution k of matching, the others is 0 , it can increase the weight of distribution, reduce weight which does not match the distribution.

The parameters will be updated for the model which match the current pixel :

$$
\begin{aligned}
& \mu_{t}=\rho I_{t}+(1-\rho) \mu_{t-1} \\
& \delta_{t}^{2}=\rho\left(I_{t}-\mu_{t}\right)^{T}\left(I_{t}-\mu_{t}\right)+(1-\rho) \delta_{t-1}^{2}
\end{aligned}
$$

The $\rho$ is the other learning rate, and it's value is ${ }^{\rho=\alpha \eta\left(\frac{I_{t}}{\mu_{k}}, \delta_{k}\right)}, \eta$ is density function of Gaussian. It remain unchanged if don't have matched distributes.

Adaptive threshold of OTSU. Because of the some pixels of foreground image which is binarization by fixed threshold in can not get accurate result. And although the overall effect can meet the requirements ,but the processing of detail is not ideal. So we introduce OTSU ${ }^{[11]}$ to look for the threshold which satisfy maximum-between-clusters-variance, and it will get best divisional effect. The main idea is the gray of picture whose level is $1 \sim \mathrm{m}$, the picture is divided two groups of 
background and foreground. The percentage points of foreground is $p_{0}$, the mean grey is $l_{0}$; the percentage points of foreground is $p_{1}$, the mean grey is $l_{1}$.the mean of all gray is :

$$
l=p_{0} \times l_{0}+p_{1} \times l_{1}
$$

Then

$$
v=p_{0} \times\left(l_{0}-l\right)^{2}+p_{1} \times\left(l_{1}-l\right)^{2}
$$

The $\mathrm{t}$ is best threshold of segmentation with traversal method which make ${ }^{v}$ maximum.

\section{The realization which combine GMM with OTSU}

\section{Implementation steps.}

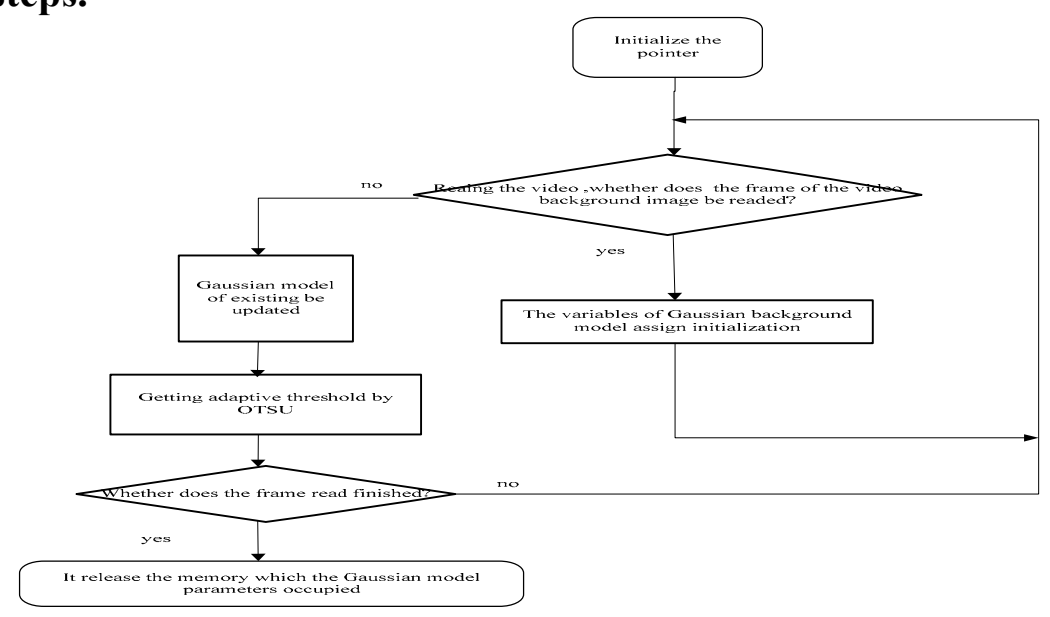

Figure 1. The implementation process

Testing result and analysis. We test program with $\mathrm{VC}++6.0$ and $\mathrm{OpenCV}$, selecting video of collection for test, the result of test as shown in Figure 1.

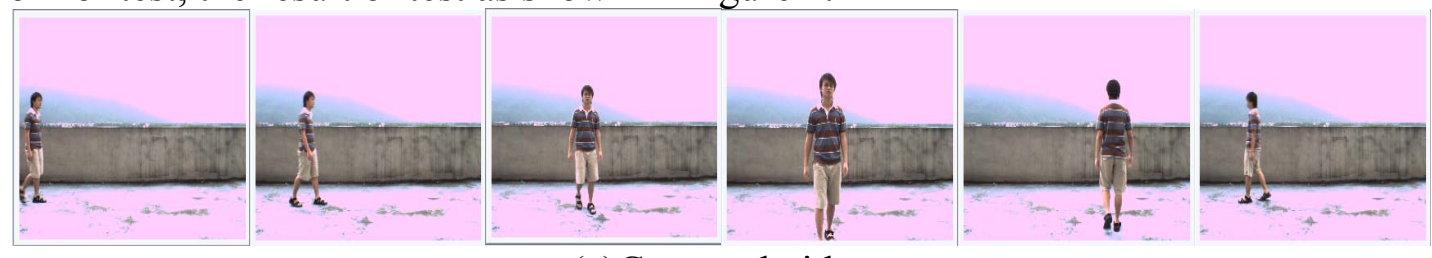

(a)Captured video



(b) Background of GMM modeling
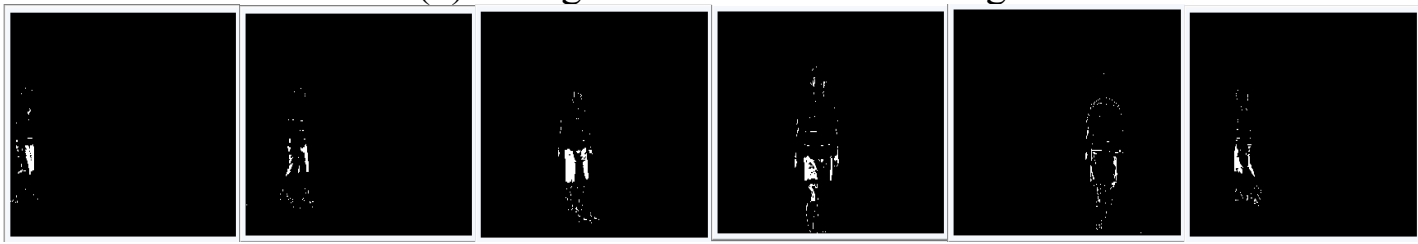

(c) Motion target of GMM detection
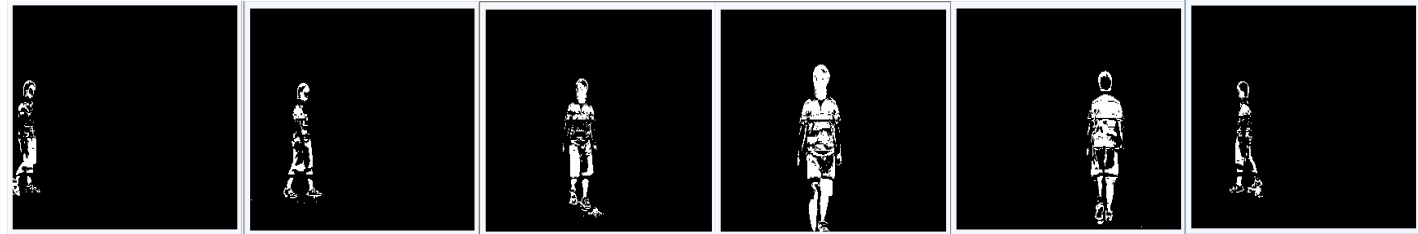

(d) Motion target of GMM unties with OTSU

Figure 2 Motion target comparison picture of GMM unties with OTSU 
The captures is video of motionless gather, and the main target of detection is moving object. Figure 1(b) and Figure 1(c) show that GMM background modeling have a good sensitive and produce less disturbing in motion object region. It was shown that the overall effect can meet the requirements from Figure 1(c) ,but the processing of detail is not ideal.

\section{Summary}

This paper propose a algorithm of moving target detection which combine with Gaussian Mixture Modeling and OTSU under studying moving object detection algorithm on readitional static camera . Because of some pixels of foreground image which is binarization by fixed threshold can not get accurate result. And although the overall effect can meet the requirements, but the processing of detail is not ideal. So we introduce OTSU to look for the threshold which satisfy maximum-between-clusters-variance, and it will get best divisional effect. The paper combined with the GMM and OTSU by technology of OpenCV. The experimental results show that moving target detection of Gaussian Mixture Model have a good effect on adaptive threshold of OTSU. It is a beginning for the detective target processing by adaptive threshold of OTSU. We will use the method this paper work out in future research work about motion target detection of Gaussian Mixture Modeling.

\section{References}

[1] Chen Jie, Wang Zhenhua, Dou Lihua. Scale Adaptive Canny Edge Detection Method[J]. OPTO-ELECTRONIC ENGINEERING, 2008, 35(2) : 79-84.

[2] Chen Dan, Zhang Feng, He Guiming. An Improved Binarization Algorithm for Document Image [J] . COMPUTER ENGINEER, 2003, 29( 13): 85-86.

[3] Xue Hongwei, Han Huilian. Study on Selective Enhancement Algorithms of Text in Multicolor Image [J]. COMPUTER AND DIGITAL ENGINEERING, 2009, 37( 4) : 124-127

[4] TONG Lijing, CHEN Kan, FU Xiaoling, DUAN Jianyong. VFCM: binarization algorithm for document image [J]. COMPUTER ENGINEERING AND DESIGN, 2009, 30( 13):3216-3218.

[5] Tong L J, Chen K, Zhang Y,et al. Document image binarization based on NFCM[J] Proc 2ndIEEE Conf Image and Signal Processing, IEEE Press, Dec.2009: 1769 -1773.

[6] STAUFFER C, GRIMSON W E L. Learning patterns of activity using real-time tracking[J]. IEEE Trans on Pattern Analysis and Ma-chine Intelligence, 2000, 22(8): 747-757.

[7] ZHANG J, CHEN C H. Moving objects detection and segmentation in dynamic video backgrounds $[\mathrm{C}] /$ /Proc of IEEE Conference on Tech-nologies for Homeland Security. 2007: 64-69

[8] YAO Hui, SU Songzhi, WANG Li, LI Shaozi. Moving Objects Detection Method Based on the Improved Adaptive Background Mixture Model [J] . JOURNAL OF XIAMEN UNIVERSITY: NATURAL SCIENCE,2008( 47): 505-510.

[9] CHEN Zujue, CHEN Xiaojun, HE Hong. Moving Object Detection Based on Improved Mixture Gaussian Models[ J]. JOURNAL OF IMAGE AND GRAPHICS, 2007( 12):1585-1589.

[10]SHIMADA A,ARITA D,TANIGUCHI R I. Dynamic control of adaptive mixture-of-Gaussians background model[C]/Proc of IEEE International Conference on Video and Signal Based Surveillance. Washington DC:IEEE Computer Society,2006:1-5.

[11]OSTU N. A threshold selection method from gray-level histograms[J].IEEE Trans. Systems, Man, and Cybernetics, 1979,9 ( 1) :62-66. 